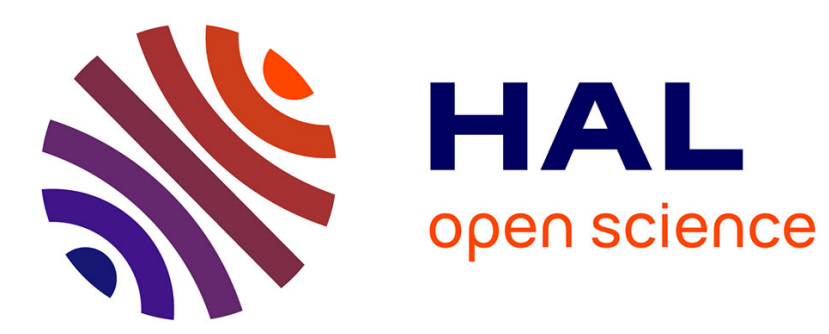

\title{
A dynamic based path tracking controller for a fast rover with independent steering and drive
}

\author{
Mohamed Krid, Faïz Ben Amar
}

\section{To cite this version:}

Mohamed Krid, Faïz Ben Amar. A dynamic based path tracking controller for a fast rover with independent steering and drive. 14th International Conference on Climbing and Walking Robots and the Support Technologies for Mobile Machines, Sep 2011, Paris, France. pp.78-85, 10.1142/9789814374286_0009. hal-03177948

\section{HAL Id: hal-03177948 \\ https://hal.science/hal-03177948}

Submitted on 24 Mar 2021

HAL is a multi-disciplinary open access archive for the deposit and dissemination of scientific research documents, whether they are published or not. The documents may come from teaching and research institutions in France or abroad, or from public or private research centers.
L'archive ouverte pluridisciplinaire HAL, est destinée au dépôt et à la diffusion de documents scientifiques de niveau recherche, publiés ou non, émanant des établissements d'enseignement et de recherche français ou étrangers, des laboratoires publics ou privés. 


\title{
A dynamic based path tracking controller for a fast rover with independent steering and drive
}

\author{
M. KRID* and F. BEN AMAR** \\ ISIR - Institut des Systèmes Intelligents et Robotiques, \\ Universit Pierre et Marie Curie Paris 6, CNRS UMR 7222 \\ PARIS, 75252 cedex 05, France \\ *E-mail : krid@isir.upmc.fr \\ **E-mail : amar@isir.upmc.fr
}

\begin{abstract}
The paper presents a path tracking controller for a fast rover which has independent front and rear steering and four independent driven wheels. The paper develop first the dynamic model of the vehicle moving on a horizontal plane. Next, the model is linearized by assuming that the side slip angles remain small and a linear lateral slippage model introducing the lateral contact stiffness is used. The kinematics of the vehicle is also linearized by assuming that the rover remains close a reference path and with a small relative heading angle. The linearized model permits the design of a Linear Quadratic Regulator LQR which is tested by using Matlab-Simulink/Adams co-simulation. Simulation results, obtained with numerous reference paths, show a good control accuracy and appears to be robust with respect to environment and robot state changes.
\end{abstract}

Keywords: Mobile robot, path tracking, wheel slippage, LQR controller.

\section{Introduction}

Outdoor mobile robots has to perform operations more and more far and more and more quickly. Therefore we are interested in the design and control of fast rovers which are able to move autonomously in natural environment at high velocity (up to $10 \mathrm{~m} / \mathrm{s}$ ) following a reference path referred by RTK-GPS points. Generally, vehicle dynamics is neglected when designing path tracking controller ${ }^{1},{ }^{2}$ however purely kinematic based approaches are clearly incongruous for solving the problem of motion control of fast rovers.

Many researches focused on path following of autonomous vehicle in presence of sliding have been developed ${ }^{1}{ }^{3}$ A kinematic model was used in ${ }^{4}$ where sliding effects are introduced as additive unknown parameters. The sliding angle was estimated on-line. Robust adaptive controller was desi- 
gned in ${ }^{5}$ and a sliding mode control is defined in. ${ }^{6}$ High-gain controller was designed in ${ }^{7}$ to solve the trajectory tracking problem. A fuzzy controller was developed in ${ }^{8}$ where several levels of competences and behaviors are implemented. These methods, as well as developed $\mathrm{in}^{2}{ }^{4}$ utilize wheel velocities and/or steering angles as control inputs, and a kinematic model of the vehicle for designing a controller which stabilizes some tracking errors. Since the vehicle dynamics is neglected, these approaches reduce hugely the control performance (accuracy, stability, robustness...).

The paper develops a dynamic model which takes into consideration the lateral slippage of wheels, the wheel torques distribution and independent front and rear steering. This paper is organized as follow. Section II starts with a description of the vehicle dynamic model with 3-DOF and using a linear slippage model. Then, the vehicle kinematic model is presented, introducing the relative tracking errors with respect to a reference path. Finally, we combine the two kinematic and dynamic models, thus providing a global linear state model. This model is used for designing a feedback controller based on quadratic optimization. This is developed in section III. The simulation results are given in the last section IV, including the performance of the proposed tracking controller.

\section{Modeling}

This section describes the vehicle dynamics model which will be used for control design. We consider the double tracks model (handling model) illustrated in Figure (1). The vehicle has 3 DOF : longitudinal, lateral and yaw motions ${ }^{9}$ of the chassis. The dynamic model is given by the following equation of motion.

$$
\begin{aligned}
\dot{V}_{x} M & =M V_{\psi} V_{y}+\left(F_{x 1}+F_{x 2}\right) \cos \beta_{f}+\left(F_{x 3}+F_{x 4}\right) \cos \beta_{r} \\
& -\left(F_{y 1}+F_{y 2}\right) \sin \beta_{f}-\left(F_{y 3}+F_{y 4}\right) \sin \beta_{r} \\
\dot{V}_{y} M & =-M V_{\psi} V_{x}+\left(F_{x 1}+F_{x 2}\right) \sin \beta_{f}+\left(F_{x 3}+F_{x 4}\right) \sin \beta_{r} \\
& +\left(F_{y 1}+F_{y 2}\right) \cos \beta_{f}+\left(F_{y 3}+F_{y 4}\right) \cos \beta_{r} \\
\ddot{\psi} I_{z} & =a\left(\left(F_{x 1}+F_{x 2}\right) \sin \beta_{f}+\left(F_{y 1}+F_{y 2}\right) \cos \beta_{f}\right) \\
& +d\left(\left(F_{x 2}-F_{x 1}\right) \cos \beta_{f}+\left(F_{y 1}-F_{y 2}\right) \sin \beta_{f}\right) \\
& -b\left(\left(F_{x 3}+F_{x 4}\right) \sin \beta_{r}+\left(F_{y 3}+F_{y 4}\right) \cos \beta_{r}\right) \\
& +d\left(\left(F_{x 4}-F_{x 3}\right) \cos \beta_{r}+\left(F_{y 3}-F_{y 4}\right) \sin \beta_{r}\right)
\end{aligned}
$$

We denote by $F_{x}$ and $F_{y}$ the longitudinal and lateral tire forces, $V_{x}$ and 
$V_{y}$ are the longitudinal and lateral velocities of the chassis center of mass, $\dot{\psi}$ is the yaw rate, $I_{z}$ is the yaw-inertia moment, $M$ is the car mass, $d$ is the half-width of the wheel-base, $a$ and $b$ are the distances from the center of gravity to the front and rear axles, $\beta_{f}$ and $\beta_{r}$ are front and rear wheel steering angles.

\subsection{Lateral force model}

Except for the gravity and aerodynamique forces, the main external forces applied on the vehicle are produced by the tires. The lateral forces acting on the front and rear tire are assumed to be a linear function of the side-slip angle, ${ }^{10}$ i.e.

$$
F_{y_{(f, r)}}=C_{(f, r)} \alpha_{(f, r)}
$$

where $C_{f}$ and $C_{r}$ are, respectively, the tire cornering stiffness of the front and rear tires.

The side-slip angle $\alpha_{f, r}$ is defined as the angle between the wheel velocity and the longitudinal axis of the wheel itself. Assuming that side-slip angles are quite small (less than $10^{\circ}$ in practice), we can use a linear approximation, that is :

$$
\alpha_{f}=\frac{V_{y}+a \dot{\psi}}{V_{x}}-\beta_{f}, \alpha_{r}=\frac{V_{y}-b \dot{\psi}}{V_{x}}-\beta_{r}
$$

\subsection{Robot dynamics model}

We focus in this paper on the lateral dynamic and path tracking. Henceforth, the longitudinal velocity $V_{x}$ will be considered constant $\left(\dot{V}_{x}=0\right)$. Combining the double tracks model (2) and the linear tire model (4) and (5), we can write the expression for the lateral acceleration :

$$
\begin{aligned}
M \dot{V}_{y} & =2 \frac{C_{f}+C_{r}}{V_{x}} V_{y}+\left(2 \frac{a C_{f}-b C_{r}}{V_{x}}-M V_{x}\right) \dot{\psi} \\
& +\left(2 F_{x f}-2 C_{f}\right) \beta_{f}+\left(2 F_{x r}-2 C_{r}\right) \beta_{r}
\end{aligned}
$$

Angular acceleration about the yaw axis can be written by combining (3), (4) and (5)

$$
\begin{aligned}
I_{z} \ddot{\psi} & =2 \frac{a C_{f}-b C_{r}}{V_{x}} V_{y}+\left(2 \frac{a^{2} C_{f}-b^{2} C_{r}}{V_{x}}\right) \dot{\psi} \\
& +a\left(2 F_{x f}-2 C_{f}\right) \beta_{f}-b\left(2 F_{x r}-2 C_{r}\right) \beta_{r}+d\left(\Delta F_{x f}+\Delta F_{x r}\right)
\end{aligned}
$$


where $\Delta F_{x f}$ and $\Delta F_{x r}$ are the longitudinal force differences between the right and left wheels, respectively, on the front and rear axle.

Let's consider $\Delta F_{x f}$ and $\Delta F_{x r}$ as new control inputs. This leads to the the following state system :

$$
\left(\begin{array}{c}
\dot{V}_{y} \\
\ddot{\psi}
\end{array}\right)=[a]\left(\begin{array}{c}
V_{y} \\
\dot{\psi}
\end{array}\right)+[b]\left(\begin{array}{c}
\beta_{f} \\
\beta_{r} \\
\Delta F_{x f} \\
\Delta F_{x r}
\end{array}\right)
$$

where $[a]$ is $2 \times 2$ matrix and $[b]$ is $2 \times 4$ matrix.

\subsection{Robot-path kinematic model}

Figure 2 illustrates the path tracking problem considered in the current study. $\psi$ and $\psi_{\text {ref }}$ are the robot heading and the heading of the local tangent of the prescribed path with respect to a fixed arbitrary reference direction. The rate of change of $\psi_{\text {ref }}$ with respect to the curvilinear distance measured along the path defines the path curvature $k . e_{\psi}$ represents the heading error of the robot. $e_{n}$ is the lateral deviation of the robot center of mass with respect to the prescribed path.

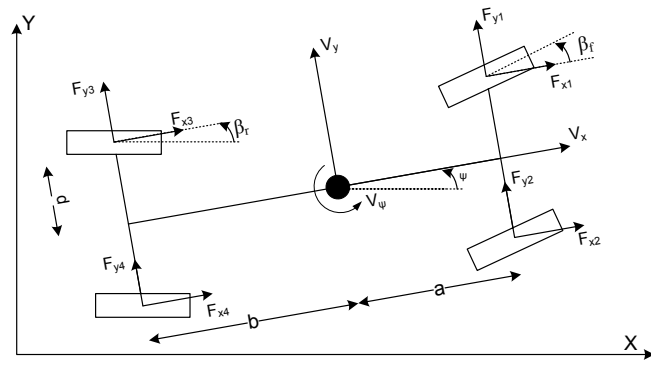

Figure 1. Double tracks model.

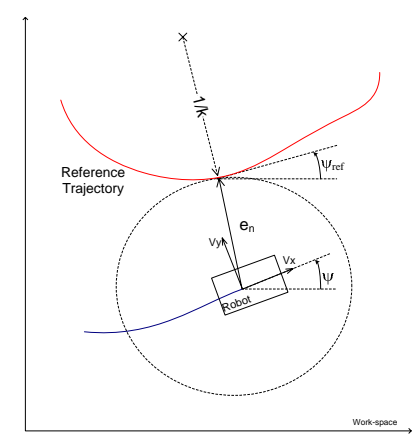

Figure 2. The reference and actual path.

Sign conventions for both $e_{\psi}$ and $e_{n}$ are denoted in Figure (2). With reference to the same figure and by assuming that the angular deviation is small, the following kinematical relationships are derived : 


$$
\begin{aligned}
\dot{e}_{n} & =V_{x} \sin e_{\psi}+V_{y} \cos e_{\psi} \\
& =V_{x} e_{\psi}+V_{y} \\
\dot{e}_{\psi}= & \dot{\psi}-\dot{\psi}_{r e f} \\
= & \dot{\psi}-\frac{V_{x} \cos e_{\psi}-V_{y} \sin e_{\psi}}{1 / k+e_{n}}
\end{aligned}
$$

In case of straight line tracking $(k=0)$, this last equation is not singular and is equivalent to $\dot{e}_{\psi}=\dot{\psi}$, since $\dot{\psi}_{\text {ref }}=0$ for a straight line path. Assuming that $e_{n}<<1 / k$, equation (10) can be linearized as follow :

$$
\dot{e}_{\psi}=\dot{\psi}-V_{x} k+V_{x} k^{2} e_{n}
$$

Assuming a constant curvature, the derivative with respect to time of this latter equation gives

$$
\ddot{e}_{\psi}=\ddot{\psi}+V_{x}^{2} k^{2} e_{\psi}+V_{x} k^{2} V_{y}
$$

\section{Design control}

In this section, we define a path tracking control of the robot using linear state feedback. Combing the kinematic model of the path-tracking errors (9) and (12) with the dynamic model of the vehicle (8) results in a new state-space model :

$$
\dot{X}=A X+B U
$$

where $X=\left[V_{y} \dot{\psi} e_{\psi} \dot{e}_{\psi} e_{n}\right]^{T}$ is the state vector and $U=$ $\left[\beta_{f} \beta_{r} \Delta F_{x f} \Delta F_{x r}\right]^{T}$ is the inputs vector, $A$ is $5 \times 5$ matrix and $B$ is $5 \times 4$ matrix.

In this study, the main objective of the control design is to improve the steering behavior of the robot in the presence of lateral and yaw disturbances. Therefore, the controller should minimizes the side-slip angle $\beta$ of the robot center mass through minimizing lateral velocity $V_{y}$ and its yaw rate (thereby decreasing the probability of lateral skidding). To achieve 
good tracking performance, the cost function must include a term that penalizes both the heading angle deviation $e_{\psi}$ and the lateral offset $e_{n}$,

$$
J=\lim _{t \rightarrow \infty} \frac{1}{t} \int_{0}^{t}\left(X^{T} Q X+U^{T} R U\right) d t
$$

The second term on the right-hand penalizes the weighted-magnitude of the control inputs vector. This will prevent the use of too high control commands and the resultant control actions will be smooth. $Q$ and $R$ are the nominal state-weighting and the input-weighting matrices.

\section{Simulation results and discussion}

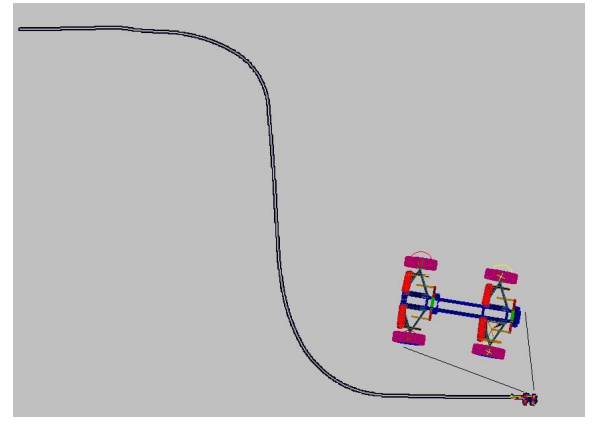

Figure $3 . \quad$ simulation views.

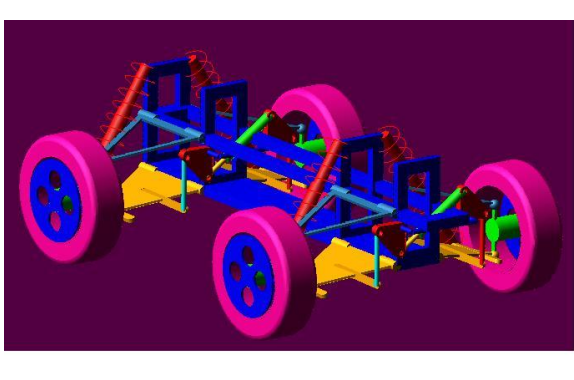

Figure 4. ADAMS views.

In this section, the LQR controller presented in the previous section has been tested using co-simulation between ADAMS for the mechanical model and Matlab for the control one. The ADAMS model consider a mechanical model with $16 \mathrm{DOF}$, including 4 independent double wishbone suspensions. The classical $Z$ and $O$ paths are used for testing the controller, they are plotted respectively on (Fig.5) and (Fig.9). In the simulation, the LQR controller gains are calculated on-line, as they depend on the longitudinal velocity $V_{x}$ and the reference path curvature $k$. The path curvature was filtered to remove the abrupt variations. During the first 3 seconds of simulation, the system is open-loop until reaching the reference longitudinal velocity. For the first path, we give the control inputs for the front and rear steering angles. The time evolution of lateral deviation and orientation errors are shown on figure (7) and figure (8). For the second path, we give 


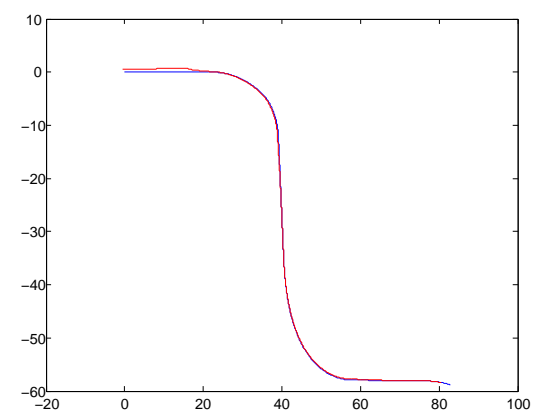

FiguRE 5. The reference (blue) and actual (red) paths for the ' $Z$ ' path.

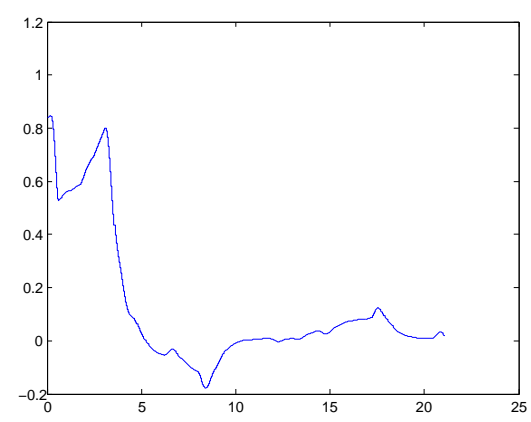

Figure 7. Lateral deviation for the ' $\mathrm{Z}$ path $[\mathrm{m}]$.

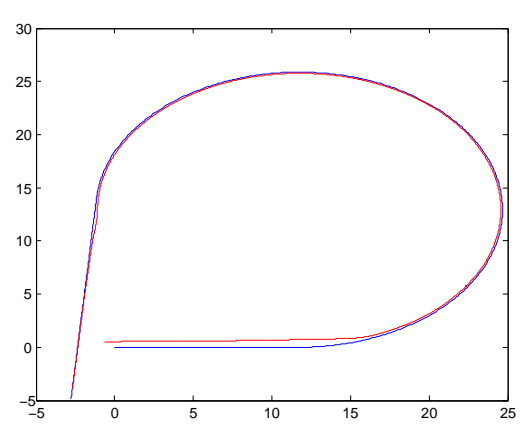

Figure 9. The reference (blue) and actual (red) paths for the 'O' path.

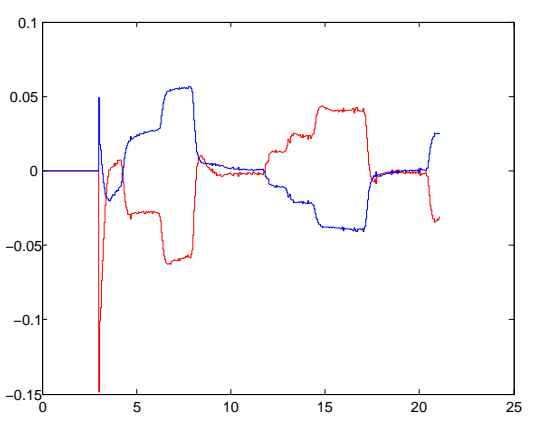

Figure 6. Steering angles : front (red) and rear (blue) for the ' $\mathrm{Z}$ ' path [rad].

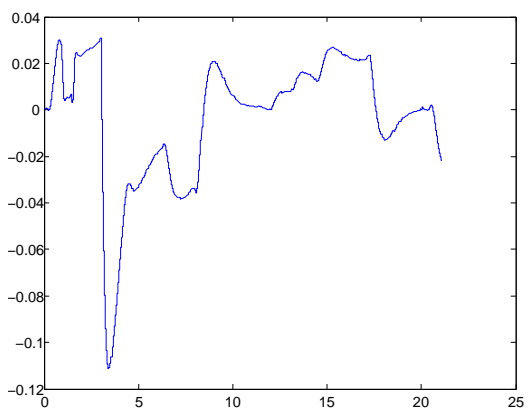

Figure 8. Yaw deviation for the 'Z' path [rad].

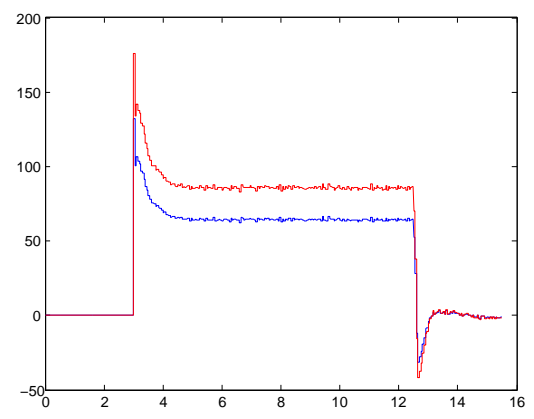

Figure 10. The differential forces on front (red) and rear (blue) axle for 'O' path $[\mathrm{N}]$. 
on figure (10) the differential forces $\Delta F_{x f} \Delta F_{x r}$ for the front and the rear axles.

Results show that the proposed LQR control based on the dynamics model is able to track the reference path with quite good accuracy and quite small and smooth inputs. The new inputs controllers, $\Delta F_{x f}$ and $\Delta F_{x r}$, seems to increase the rank of the controllability matrix what permits to reduce the steering angles.

\section{Bibliographie}

1. H. Fang, R. Lenain, B. Thuilot and P. Martinet, Trajectory tracking control of farm vehicles in presence of sliding, in Intelligent Robots and Systems, 2005. (IROS 2005). 2005 IEEE/RSJ International Conference on, aug. 2005.

2. S. L. Tan and J. Gu, Investigation of trajectory tracking control algorithms for autonomous mobile platforms : theory and simulation, in Mechatronics and Automation, 2005 IEEE International Conference, july-1 aug. 2005.

3. N. Bouton, R. Lenain, B. Thuilot and P. Martinet, Backstepping observer dedicated to tire cornering stiffness estimation : application to an all terrain vehicle and a farm tractor, in Intelligent Robots and Systems, 200\%. IROS 200\%. IEEE/RSJ International Conference on, 29 2007-nov. 22007.

4. C. Cariou, R. Lenain, B. Thuilot and P. Martinet, Adaptive control of fourwheel-steering off-road mobile robots : Application to path tracking and heading control in presence of sliding, in Intelligent Robots and Systems, 2008. IROS 2008. IEEE/RSJ International Conference on, sept. 2008.

5. H. Fang, R. Lenain, B. Thuilot and P. Martinet, Robust adaptive control of automatic guidance of farm vehicles in the presence of sliding, in Robotics and Automation, 2005. ICRA 2005. Proceedings of the 2005 IEEE International Conference on, april 2005.

6. J.-M. Yang and J.-H. Kim, Robotics and Automation, IEEE Transactions on 15, 578 (jun 1999).

7. M. L. Corradini, T. Leo and G. Orlando, International Journal of Robotic Systems 19, 177(April 2002).

8. S. Yang, H. Li, M.-H. Meng and P. Liu, Fuzzy Systems, IEEE Transactions on 12, 436 (aug. 2004).

9. D.-C. Liaw, W.-C. Chung, C.-C. Song and C.-S. Hsieh, A feedback linearization design for the control of vehicle's lateral dynamics, in SICE-ICASE, 2006. International Joint Conference, oct. 2006.

10. S. Jung and T. Hsia, Explicit lateral force control of an autonomous mobile robot with slip, in Intelligent Robots and Systems, 2005. (IROS 2005). 2005 IEEE/RSJ International Conference on, aug. 2005. 